\title{
Systemic or local thrombolysis in high-risk pulmonary embolism
}

\author{
Liviu Macovei ${ }^{1,2}$, Razvan Mihai Presura², Catalina Arsenescu Georgescu ${ }^{1,2}$ \\ ${ }^{1}$ Department of Cardiology, Cardiovascular Diseases Institute "Prof. Dr. George I M Georgescu”, Romania \\ ${ }^{2}$ University of Medicine and Pharmacy "Gr. T. Popa", Iasi, Romania
}

\begin{abstract}
Background and aim: High-risk pulmonary embolism (PE) represents an important health problem in emergency cardiology, being associated with a high rate of mortality. The aim of this study is to assess the efficacy and safety of pulmonary intra-arterial thrombolysis with streptokinase compared to systemic thrombolysis.

Methods and Results: In our study, 28 patients with acute high risk PE were treated by intra-arterial thrombolysis with clinical success rate of $96.4 \%$, while in the group with systemic thrombolysis (24 patients) the rate of clinical success was significantly lower (70.8\%). Also, pressure gradient between right ventricle $(R V)$ and right atrial $(R A)\left(P_{R V-R A}\right)$ decreased significantly in patients treated by pulmonary intra-arterial thrombolysis instead of systemic thrombolysis. Mortality during the hospitalization was $0 \%$ in the group with local thrombolysis and $29.2 \%$ in the other group, with a significant statistical difference. Major bleeding complications appeared in $14.3 \%$ of the patients with local thrombolysis and in $20.8 \%$ of the ones treated by systemic thrombolysis, without statistical significance. Moreover, the proportion of minor bleeding was comparable in the two groups of patients. There was no intracranial bleeding. Disseminated intravascular coagulation occurred in 1 patient with systemic thrombolysis.

Conclusions: The rate of clinical success and the regression of $R V$ overload were significantly higher in patients treated by pulmonary intra-arterial thrombolysis. The results regarding the efficiency of the pulmonary intra-arterial thrombolysis in high-risk PE are encouraging, the mortality in these patients being significantly lower than the one for systemic administration of the thrombolytic agent. (Cardiol J 2015; 22, 4: 467-474)
\end{abstract}

Key words: pulmonary embolism, local thrombolysis

\section{Introduction}

High-risk pulmonary embolism (PE) represents an important health problem in emergency cardiology, being associated with a high rate of mortality [1].

If the early mortality after PE with a high risk is due to arterial hypotension, afterwards, the major cause of death is represented by acute right cardiac failure, the mortality after 3 months being approximately 50\% [1-3]. In the International Cooperative Pulmonary Embolism Registry, the 90-day mortality rate for patients with acute $\mathrm{PE}$ and systolic blood pressure $<90 \mathrm{~mm} \mathrm{Hg}$ was $52.4 \%$ $[4,5]$. In the Germany-based Management Strategy and Prognosis of Pulmonary Embolism Registry, in-hospital mortality was $25 \%$ for those presenting with cardiogenic shock $[4,6]$.

Survival in high risk PE depends on the success of the therapy of pulmonary revasculariza-

Address for correspondence: Dr Liviu Macovei, Cardiovascular Diseases Institute, Iasi, Carol I Bld, nr 50, 700503, Romania, e-mail: liviughemacovei@yahoo.com 
tion. Studies demonstrated that patients receiving thrombolytic therapy had more rapid resolution of angiographic obstruction and hemodynamic disturbances than those receiving heparin alone [7-9].

In 1997, the Food and Drug Administration approved the use of thrombolytic therapy for PE associated with hypotension and significant hypoxemia despite oxygen supplementation $[7,10]$. One of the actual controversies of the thrombolytic therapy for $\mathrm{PE}$ is which thrombolytic agent is the most effective and should the agent be administered systemically or locally? Many studies demonstrated that streptokinase (SK), urokinase and recombinant tissue plasminogen activator have equal safety profiles and efficacy in angiographic resolution of clot and improvement of hemodynamic disturbances [7, 11].

An alternative to systemic thrombolysis is represented by pulmonary intra-arterial thrombolysis, however there is still a continuous controversy regarding superior benefits yet with lower risk of bleeding. There are few situations that suggest a superior efficiency of pulmonary intra-arterial thrombolysis in high risk PE and the thrombolytic agents that are used are the following: tissue plasminogen activator, monoteplase, urokinase [2].

In our study, we used SK as thrombolytic agent administrated systemic in one group of patients and pulmonary intra-arterial in the other group. The results regarding the efficiency of the pulmonary intra-arterial thrombolysis in $\mathrm{PE}$ with high risk are encouraging, the mortality in these patients being significantly lower than systemic administration of the thrombolytic agent.

The aim of this study is to assess the efficacy and safety of pulmonary intra-arterial thrombolysis with SK compared with systemic thrombolysis.

\section{Methods}

\section{Study population}

The patients admitted to the study have accomplished the following criteria of inclusion: (1) Diagnosis of high risk PE; (2) Informed consent of the patient regarding the treatment.

\section{Study protocol}

Exclusion criteria were represented by: hemorrhagic stroke or stroke of another unknown etiology somewhere in the past, ischemic stroke in the last 6 months, lesions or cancer of the central nervous system, major trauma, surgery or recent cranial trauma (the last 3 weeks), gastrointestinal bleeding in the last month, known bleeding, and pregnancy.
All the echocardiographic and angiographic studies have been performed in the same laboratories using the same contrast agent and the same protocol of diagnosis.

The modality of giving the thrombolytic agent, systemic or intrapulmonary, was randomly chosen. The thrombolytic agent was: SK 250,000 UI/30 min followed by $100,000 \mathrm{UI} / \mathrm{h}$. The adjuvant therapy was: anticoagulants (unfractionated heparin, lowmolecular-weight heparin), antiplatelet agents (clopidogrel, aspirin) and their associations.

The followed parameters, at the admission in the intensive care unit and at the end of the treatment, have been grouped as follows: clinical features, hemodynamic parameters, electrocardiographic and angiographic aspects. Clinical evolution was evaluated by the presence of dyspnea, chest pain, syncope and asymptomatic status at the end of the treatment. Hemodynamic parameters were: systolic arterial blood pressure less than $90 \mathrm{~mm} \mathrm{Hg}$, peripheral oxygen saturation and heart rate. The electrocardiographic study was constant in the followed evolution of the axis of QRS complex (A QRS), reversibility of right bundle block, S1Q3T3 aspect, negative $T$ waves from $V_{1}$ up to $V_{3}$ and the atrial arrhythmias. The followed echocardiographic parameters were: telediastolic diameter of right ventricular (RVD2), lateromedial diameter of right atrium (RA minor-axis), pressure gradient between right ventricle $(\mathrm{RV})$ and right atrium $(\mathrm{RA})\left(\mathrm{P}_{\mathrm{RV}-\mathrm{RA}}\right)$, right bundle's diameter of the right pulmonary artery (RPA) and the inferior cave vein's diameter (IVC). The evolution of the mean pressure in the pulmonary artery (mean PAP) was monitored angiographically. The valid patients were represented by those who had their parameters valid before and after the thrombolysis was performed.

The efficiency and safety profile of the used treatment has been evaluated by monitoring the complete thrombolysis (evaluated angiographic and/or echocardiographic), the appearance of major bleeding complications (brain bleeding or gastrointestinal, massive hematoma on the puncture site or acute post-hemorrhagic anemia) or minor complications (minor bleeding, hematoma on the puncture site, transient ischemic attack) and disseminated intravascular coagulation (CID).

\section{Statistical analysis}

The centralization of the data was done nominally and numerically. Nominal data was characterized by absolute and relative frequency. Numerical data have been studied from the view of the parameters of the central tendency (media and median) 
and the dispersion (standard deviation, minimum, maximum, rank, quartiles).

Intergroup statistical data were analyzed through the nominal variables using Fisher's exact test. In the case of numeric variables, the distribution of data was determined by using Shapiro-Wilk's test. The not normal distribution of data imposed the use of nonparametric Mann-Whitney U test. The level of statistical significance was 0.05 for all tests that have been used.

Statistical analysis of the data was performed with SPSS Statistics version 17.

\section{Results}

Fifty-two consecutive patients included in the study were randomized in two groups of treatment: the administration of SK directly in the pulmonary artery (in situ) in 28 patients and systemic (intravenous) in 24 patients. The characteristics of the patients at the initial moment of the study are presented in Table 1.

\section{Clinical results}

At the end of the study, 27 (96.4\%) patients with in situ thrombolysis were asymptomatic, compared with $17(70.8 \%)$ patients with systemic thrombolysis. Statistical analysis showed a significant statistical difference between the two groups $(p=0.018$; exact sig $[2$-sided $])$.

\section{Hemodynamic findings}

The evaluation of hemodynamic parameters, before and after thrombolysis, confirmed a different evolution in the two groups. Cardiogenic shock initially identified in 18 patients, the same in both groups, has totally remitted in the group with thrombolysis in situ, meanwhile, in the group with systemic thrombolysis, it still remained in $7(29.2 \%)$ patients $(\mathrm{p}<0.03)$. The improvement of $\mathrm{SO}_{2}$ was registered in both groups, without significant statistical differences $(\mathrm{p}<0.017)$. Heart rate has dropped in both groups, in thrombolysis in situ from a median of $100 \mathrm{bpm}$ to $70 \mathrm{bpm} \mathrm{(32 \% )}$ and in systemic thrombolysis from a median of 100 bpm to $75 \mathrm{bpm}(15.5 \%)$, with registered significant statistical difference.

\section{Electrocardiographic results}

Regarding transient right bundle branch block, $\mathrm{S}_{1} \mathrm{Q}_{3} \mathrm{~T}_{3}$, negative $\mathrm{T}$ wave in $\mathrm{V}_{1}-\mathrm{V}_{3}$, and atrial arrhythmias there were no significant differences between groups.
Table 1. Selected baseline characteristics of patients.

\begin{tabular}{|c|c|c|}
\hline & \multicolumn{2}{|c|}{$\begin{array}{l}\text { Route of thrombolytic } \\
\text { administration }\end{array}$} \\
\hline & In situ & $\begin{array}{l}\text { Systemic } \\
\text { vein }\end{array}$ \\
\hline $\begin{array}{l}\text { No. of patient } \\
\text { in group }\end{array}$ & 28 & 24 \\
\hline Men/women & $13 / 15$ & $10 / 14$ \\
\hline Age [year] & $56.3 \pm 13.2$ & $532 \pm 13$ \\
\hline $\begin{array}{l}\text { Time to thrombolysis, } \\
\text { median [day] }\end{array}$ & 2.5 & 3 \\
\hline \multicolumn{3}{|c|}{ Predisposing factors for VTE: } \\
\hline Chronic heart failure & $8(28.6 \%)$ & $4(16.4 \%)$ \\
\hline $\begin{array}{l}\text { Oral contraceptive } \\
\text { therapy }\end{array}$ & $4(14.3 \%)$ & $1(4.2 \%)$ \\
\hline Malignancy & $2(7.1 \%)$ & $2(8.3 \%)$ \\
\hline Previous VTE & $8(28.6 \%)$ & $14(53.8 \%)$ \\
\hline Bed rest $>3$ days & $4(14.3 \%)$ & $4(16.7 \%)$ \\
\hline Obesity & $17(60.7 \%)$ & $15(62.5 \%)$ \\
\hline Varicose veins & $14(50 \%)$ & $10(41.7 \%)$ \\
\hline \multicolumn{3}{|l|}{ Clinical markers: } \\
\hline Dyspnea & $28(100 \%)$ & $24(100 \%)$ \\
\hline Chest pain & $18(63.4 \%)$ & $14(53.8 \%)$ \\
\hline Syncope & $11(39.3 \%)$ & $9(37.5 \%)$ \\
\hline
\end{tabular}

VTE — venous thromboembolism

\section{Echocardiographic results}

The results of echocardiographic examination are summarized in Table 2. RVD2, in thrombolysis in situ reduced from a maximum of $52 \mathrm{~mm}$ (range $22 \mathrm{~mm}$ ) to a maximum of $41 \mathrm{~mm}$ (range $14 \mathrm{~mm}$ ), median decreased only with $7 \%$, presenting a significant statistical difference $(\mathrm{p}=0.036$; exact sig. 1-tailed). In systemic thrombolysis, 7 patients (deceased, from whom 3 with bleeding complication) did not present improvement of RVD2, compared to thrombolysis in situ, where only in 2 patients the RVD2 did not decrease (Fig. 1).

RA minor-axis was reduced in both types of thrombolysis. Secondary to the in situ administration, RA minor-axis dropped from a maximum of $57 \mathrm{~mm}$ (range $24 \mathrm{~mm}$ ) to a maximum of $48 \mathrm{~mm}$ (range $24 \mathrm{~mm}$ ), median decreasing by $14 \%$. In systemic administration, RA minor-axis dropped from a maximum of $67 \mathrm{~mm}$ (range $32 \mathrm{~mm}$ ) to a maximum of $66 \mathrm{~mm}$ (range of $36 \mathrm{~mm}$ ), median decreasing by only $3 \%$, representing a significant statistical difference ( $p=0.009$; exact sig. 1-tailed). The 
Table 2. Echocardiographic parameters.

\begin{tabular}{lcc} 
& \multicolumn{2}{c}{$\begin{array}{c}\text { Route of thrombolytic } \\
\text { administration }\end{array}$} \\
\cline { 2 - 3 } & In situ & Systemic vein \\
\hline RVD2 (median): & & \\
Before [mm] & 42 & 43 \\
After [mm] & 32 & 36 \\
PD [\%] & $22^{\mathrm{A}}$ & $7^{\mathrm{A}}$ \\
RA minor-axis (median): & \\
Before [mm] & 47 & 46 \\
After [mm] & 40 & 45 \\
PD [\%] & $14^{\mathrm{B}}$ & $3^{\mathrm{B}}$ \\
P & & \\
B-RA (median): & & 49 \\
After [mm Hg] & 48 & 32 \\
PD [\%] & 26 & $35^{\mathrm{C}}$ \\
IVC (median): & $43.5^{\mathrm{C}}$ & \\
Before [mm] & & 27.5 \\
After [mm] & 21.5 & $0^{\mathrm{E}}$ \\
PD [\%] & 17 & 20.5 \\
RPA (median): & $25^{\mathrm{D}}$ & $4^{\mathrm{D}}$ \\
Before [mm] & & \\
After [mm] & 27.5 & 25 \\
PD [\%] & $14^{\mathrm{E}}$ & \\
\hline
\end{tabular}

${ }^{A} p=0.036 ;{ }^{B} p=0.009 ;{ }^{C} p=0.038 ;{ }^{D} p=0.002 ;{ }^{E} p=0.027$ : significance of difference between the two groups after the thrombolysis (exact sig. [1-tailed]); PD - percentage difference; RVD2 - telediastolic diameter of right ventricular; RA minor-axis - lateromedial diameter of right atrium; $P_{\mathrm{RV}-\mathrm{BA}}$ - pressure gradient between right ventricle and right atrium; IVC - inferior cave vein's diameter; RPA - right bundle's diameter of the right pulmonary artery

lack of regression of RA minor-axis was recorded on 8 patients with systemic thrombolysis, from whom 7 deceased (1 major bleeding complication and 3 with minor bleeding) and on 3 patients with thrombolysis in situ with minor bleeding complication (Fig. 1).

$\mathrm{P}_{\mathrm{RV}-\mathrm{RA}}$ had different variation in the two modalities of administration of the thrombolytic agent. In the case of thrombolysis in situ, the maximum dropped from $80 \mathrm{~mm} \mathrm{Hg}$ (range $75 \mathrm{~mm} \mathrm{Hg}$ ) to $70 \mathrm{~mm} \mathrm{Hg}$ (range $60 \mathrm{~mm} \mathrm{Hg}$ ), compared with systemic thrombolysis where the maximum remained $100 \mathrm{~mm} \mathrm{Hg}$, but the range increased from $75 \mathrm{~mm} \mathrm{Hg}$ before thrombolysis to $85 \mathrm{~mm} \mathrm{Hg}$ at the end of the treatment. The analysis of percentage reduction of $\mathrm{P}_{\mathrm{RV}-\mathrm{RA}}$, depending on the modality of administration of thrombolytic agent, showed a reduction by $43.5 \%$ in thrombolysis in situ and by $35 \%$ in systemic thrombolysis, showing a significant statistical dif- ference ( $\mathrm{p}=0.036$; exact sig. 1-tailed). Maintaining the same value of $\mathrm{P}_{\mathrm{RV}-\mathrm{RA}}$, it was registered only in the group with systemic thrombolysis in 6 patients (deceased patients, from whom 1 with major bleeding and 3 with minor bleeding) (Fig. 2).

Decreasing of IVC diameter was registered both in the case of thrombolysis in situ as well as in systemic thrombolysis. In situ administration determined a reduction of IVC from a maximum of $30 \mathrm{~mm}$ (range $18 \mathrm{~mm}$ ) to a maximum of $25 \mathrm{~mm}$ (range $11 \mathrm{~mm}$ ), compared with systemic administration in which IVC decreased from a maximum of $33 \mathrm{~mm}$ (range $18 \mathrm{~mm}$ ) to a maximum of $28 \mathrm{~mm}$ (range $13 \mathrm{~mm}$ ). Median IVC presented a reduction of $25 \%$ in thrombolysis in situ and a reduction of $4 \%$ in systemic thrombolysis, showing a significant statistical difference $(\mathrm{p}=0.002$; exact sig. 1 -tailed $)$. IVC remained the same in 9 patients in systemic thrombolysis group (7 deceased - out of whom 1 patient with massive bleeding and 3 with minor bleeding, and 2 surviving patients) and in 3 patients in the group with thrombolysis in situ (all 3 with minor bleeding) (Fig. 2).

The evaluation of RPA diameter before and after thrombolysis showed significant differences depending on the modality of the administration of the thrombolytic ( $\mathrm{p}=0.027$; exact sig. 1 -tailed) median RPA diameter in systemic thrombolysis having approximately the same value, compared with thrombolysis in situ in which decreased by 14\%. In thrombolysis in situ, RPA decreased from a maximum of $35 \mathrm{~mm}$ (range $13 \mathrm{~mm}$ ) to a maximum of $32 \mathrm{~mm}$ (range $13 \mathrm{~mm}$ ), compared to systemic thrombolysis in which RPA decreased from a maximum of $33 \mathrm{~mm}$ (range $11 \mathrm{~mm}$ ) to a maximum of $30 \mathrm{~mm}$ (range $11 \mathrm{~mm}$ ). RPA remained the same in 7 patients from the group with systemic thrombolysis (patients deceased, out of which 1 patient with massive bleeding and 3 with minor bleeding) and 1 patient in the group with thrombolysis in situ (Fig. 3).

The qualitative evaluation of the RV function was done by monitoring the reversibility of paradoxical movement of interventricular sept and the hypokinesia of the free wall of RV. In the group with thrombolysis in situ, from 26 patients with paradoxical movement of interventricular sept before the thrombolysis was made, the paradoxical movement was transitory, in $13(50 \%)$ patients and the hypokinesia of the free wall of RV, present at the beginning in 7 patients, was transitory in 5 of them $(71.4 \%)$. In the group with systemic thrombolysis, out of 22 patients with paradoxical movement of interventricular sept before the thrombolysis 


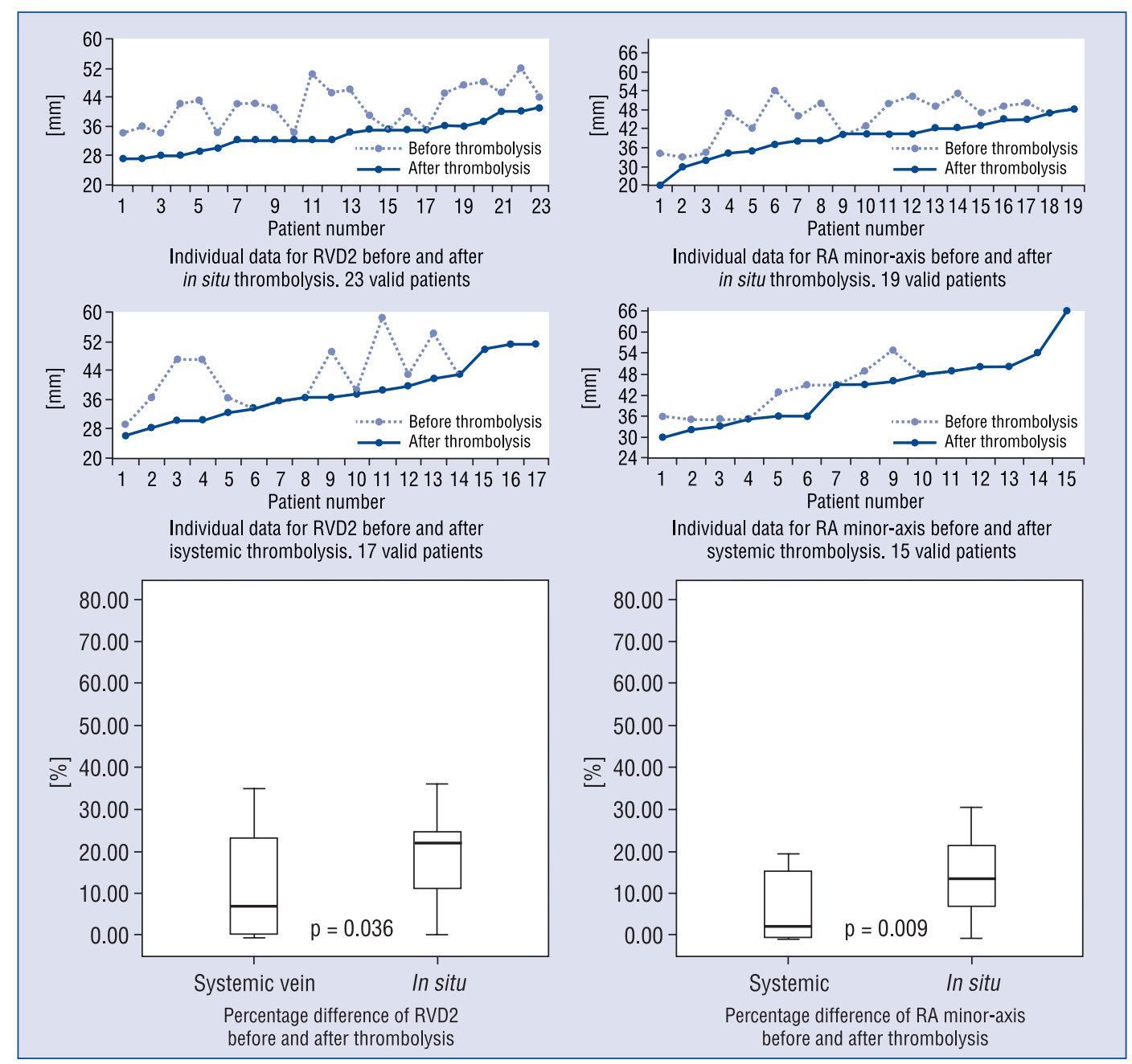

Figure 1. Individual data and percentage difference for telediastolic diameter of right ventricular (RVD2) and lateromedial diameter of right atrium (RA minor-axis) before and after thrombolysis. Individual data are ordered ascendently. Box plots express median, $25 \%$ and $75 \%$ quartiles, minimum and maximum.

was made, in $15(68.2 \%)$ it was transitory, and the hypokinesia of the free wall of RV, present at the beginning in 4 patients, was transitory in $2(50 \%)$ patients. The difference recorded in the two modalities of thrombolysis was not statistically significant neither in the case of paradoxical movement of interventricular sept $(\mathrm{p}=0.249$; exact sig. 2 -sided) nor in the case of hypokinesia of free wall of RV ( $\mathrm{p}=0.576$; exact sig. 2-sided).

\section{Angiographic finding}

The evolution of mean PAP was dependent on the mode of administration of the thrombolytic agent. In thrombolysis in situ, the mean PAP reduced from a maximum of $66 \mathrm{~mm} \mathrm{Hg}$ (range $42 \mathrm{~mm} \mathrm{Hg}$ ) to a maximum of $45 \mathrm{~mm} \mathrm{Hg}$ (range $35 \mathrm{~mm} \mathrm{Hg}$ ), the median reducing by $43 \%$. Secondary to systemic administration of thrombolytic agent, the maximal value of the mean PAP remained the same $(52 \mathrm{~mm} \mathrm{Hg}$, range $36 \mathrm{~mm} \mathrm{Hg}$ ), with the median reducing insignificantly from $50 \mathrm{~mm} \mathrm{Hg}$ to 48 mm Hg ( $>$ < 0.001; exact sig. 1-tailed) (Fig. 4).

Total lysis of the thromb (evaluated angiographic and/or echocardiographic) was objectified in $12(50 \%)$ out of 24 patients evaluated from the group with thrombolysis in situ. In the patients with systemic thrombolysis, the lysis of the entire thromb was objectified in 5 of them $(41.7 \%)$ from a total of 12 patients evaluated. Statistical analysis didn't show greater efficiency in thrombolysis in situ $(\mathrm{p}=0.454$; exact sig. 1 -sided $)$.

\section{Safety profile}

The thrombolytic agent that had been used was SK. The median of the treatment was 3 days, with limits between 1 and 4 days in thrombolysis in 


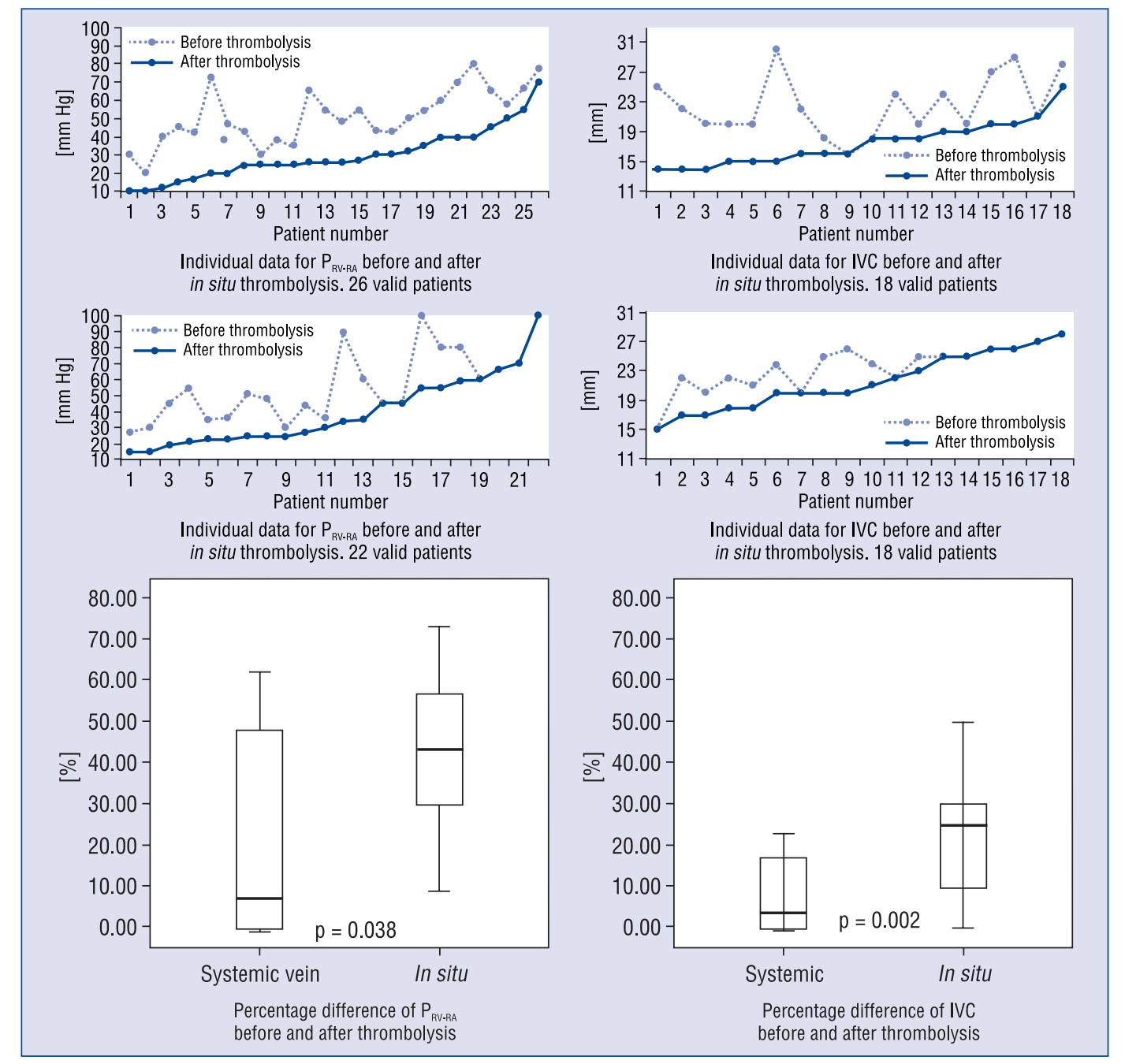

Figure 2. Individual data and percentage difference for pressure gradient between right ventricle and right atrium $\left(\mathrm{P}_{\mathrm{RV}-\mathrm{RA}}\right)$ and inferior cave vein's diameter (IVC) before and after thrombolysis. Individual data are ordered ascendently. Box plots express median, $25 \%$ and $75 \%$ quartiles, minimum and maximum.

situ, and 2 days, with limits between 1 and 3 days, in systemic thrombolysis. The statistical analysis of the duration of the thrombolytic treatment depending on the modality of the administration, showed the fact that the duration of the thrombolysis in situ was significantly longer compared with the systemic thrombolysis $(\mathrm{p}=0.002$; exact sig. 1-tailed).

The safety of the thrombolytic treatment was evaluated by analyzing the occurrence of complications. Major bleeding complications occurred in $4(14.3 \%)$ patients in the group with thrombolysis in situ, compared to 5 (20.8\%) patients in the group with systemic thrombolysis, minor bleeding being present in $6(21.4 \%)$ patients in the group with thrombolysis in situ, compared to 7 (29.2\%) patients in the group with systemic thrombolysis. CID appeared in $1(3.6 \%)$ patient with systemic thrombolysis. By analyzing the significant difference of the two ways of administration of the thrombolytic, the bleeding complications wasn't significantly more frequent in systemic thrombolysis (major bleeding, $\mathrm{p}=0.270$; exact sig. 1 -sided; minor bleeding, $\mathrm{p}=0.373$; exact sig. 1 -sided).

\section{Mortality}

Of the total number of the patients included in this study, 7 were registered as deceased, all of them from the group with systemic thrombolysis $(29.2 \%)$, the mortality being significantly higher in the patients with systemic thrombolysis $(\mathrm{p}=0.003$; exact sig. 1-sided). 


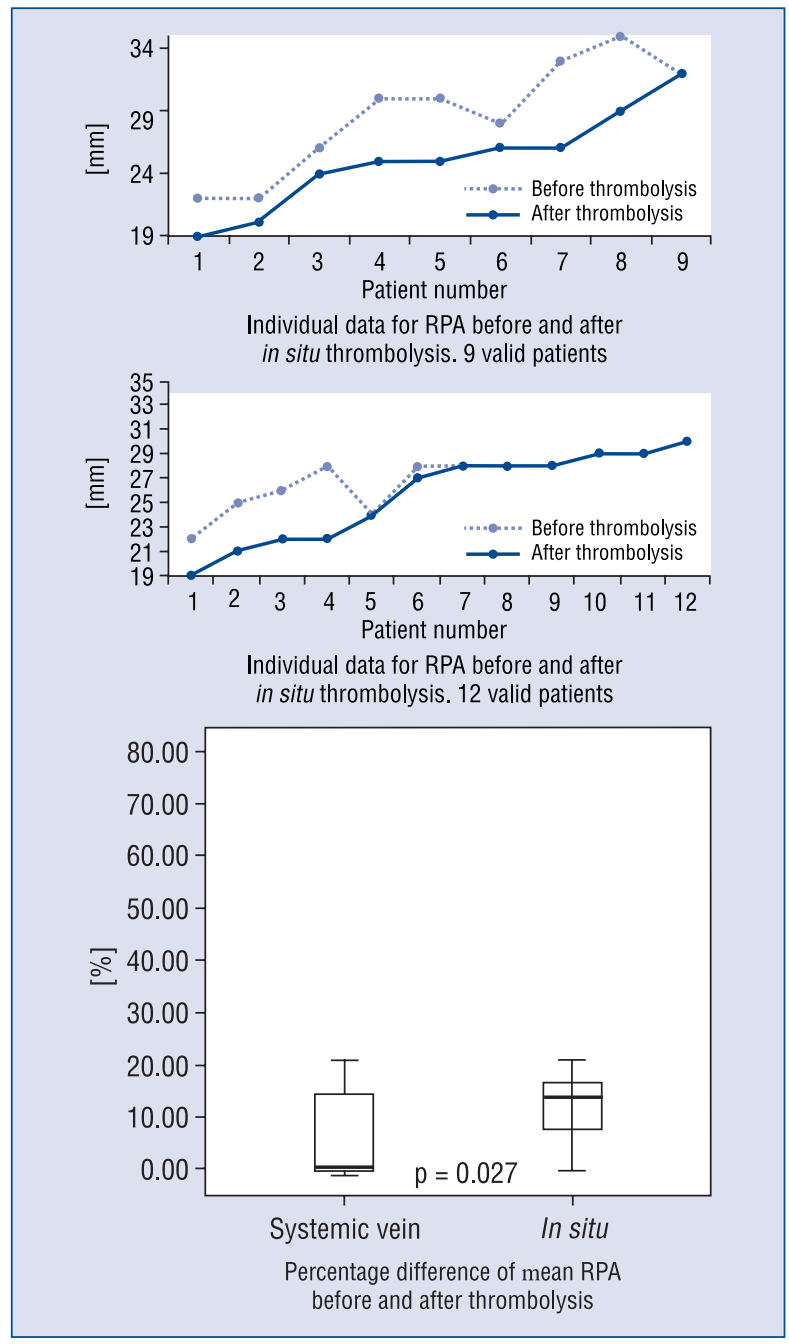

Figure 3. Individual data and percentage difference for right bundle's diameter of the right pulmonary artery (RPA) before and after thrombolysis. Individual data are ordered ascendently. Box plots express median, $25 \%$ and $75 \%$ quartiles, minimum and maximum.

\section{Discussion}

Because the patients with massive PE are at high risk of death, the American College of Chest Physicians and the European Society of Cardiology have recommended thrombolytic therapy for these patients [2].

The actual controversies are related in the first place to the method of administration of the thrombolytic agent. Thus, some of the authors do not report differences in efficiency between local and systemic administration, several reports showed that local thrombolysis improves severe hemodynamic impairment rapidly and more safety $[12,13]$.

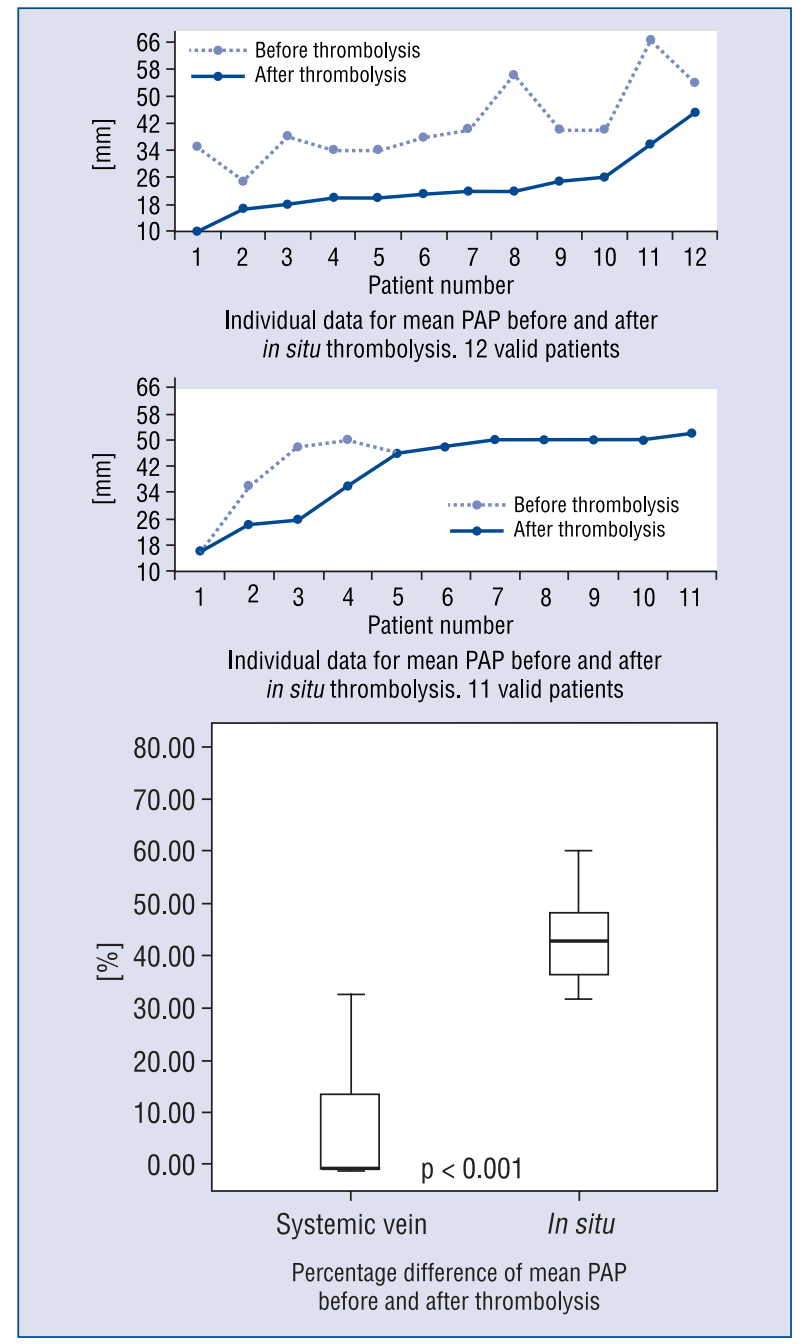

Figure 4. Individual data and percentage difference for mean pressure in the pulmonary artery (mean PAP) before and after thrombolysis. Individual data are ordered ascendently. Box plots express median, $25 \%$ and $75 \%$ quartiles, minimum and maximum.

In our study, 28 patients with acute high risk $\mathrm{PE}$ were treated by intra-arterial thrombolysis, with a clinical success rate of $96.4 \%$, the results being superior to the previous review $[2,13]$. The rate of clinical success in patients with systemic thrombolysis was significantly lower (70.8\%). Regression of RV overload after local thrombolysis in $\mathrm{PE}$ is associated with reduced morbidity and mortality [14]. In our study, $\mathrm{P}_{\mathrm{RV}-\mathrm{RA}}$ has decreased significantly more in patients treated by pulmonary intra-arterial thrombolysis instead of systemic thrombolysis.

Reported complications associated with local thrombolysis include RV perforation, significant bleeding at the puncture site, mechanical hemoly- 
sis and blood loss [2, 15-17]. Among 304 patients from the International Cooperative Pulmonary Embolism Registry (ICOPER) who received PE thrombolysis, $21.7 \%$ suffered major bleeding and $3 \%$ had intracranial bleeding [18].

Major bleeding complications appeared in our study in $14.3 \%$ of the patients with high risk PE with in situ thrombolysis and in $20.8 \%$ of the ones treated by systemic thrombolysis, but without statistical significance. There was no intracranial bleeding present in our study.

Also, the proportion of minor bleeding was comparable in the two groups of patients. Disseminated intravascular coagulation occurred in 1 patient with systemic thrombolysis.

The most impressive result was the one regarding the mortality during the hospitalization, which was $0 \%$ in the group with local thrombolysis with SK and $29.2 \%$ in the group with systemic thrombolysis, with a significant statistical difference. The surviving of all the patients with $\mathrm{PE}$ with high risk who had pulmonary intra-arterial thrombolysis was remarkable, the results being superior to the ones registered in previous situations $[2,18]$.

The present study possesses some limitations regarding non-randomized study, that is relatively small samples.

\section{Conclusions}

Local thrombolysis in high risk PE improves severe hemodynamic impairment rapidly and safety. In situ thrombolysis has a significantly higher rate of clinical success, which reduces morbidity and mortality.

\section{Conflict of interest: None declared}

\section{References}

1. Todoran TM, Sobieszczyk P. Catheter-based therapies for massive pulmonary embolism. Progress Cardiovasc Diseases, 2010; 52: 429-437.

2. Ina Y, Kondo K, Iwabuchi M. Local thrombolysis for acute massive pulmonary embolism using a pulse-infusion-thrombolysis catheter. Yonago Acta Medica, 2010; 53: 17-24.
3. Kucher N. Catheter interventions in massive pulmonary embolism. Cardiol Rounds, 2006; 10: 3.

4. Jaff MR, McMurtry MS, Archer SL et al. Management of massive and submassive pulmonary embolism, iliofemoral deep vein thrombosis, and chronic thromboembolic pulmonary hypertension: A scientific statement from the Americam Heart Association. Circulation, 2011; 123: 1788-1830.

5. Kucher N, Rossi E, De Rosa M, Goldhaber SZ. Massive pulmonary embolism. Circulation, 2006; 113: 577-582.

6. Kasper W, Konstantinides S, Geibel A et al. Management strategies and determinants of outcome in acute major pulmonary embolism: results of a multicentric registry. J Am Coll Cardiol, 1997; 30: 1165-1171.

7. Carlos E. Girod. PCCU (Pulmonary and Critical Care Update) Online, ACCP December 2000; Volume 14, Lesson 19.

8. The Urokinase Pulmonary Embolism Trial. JAMA, 1970; 214: 2163-2172.

9. Urokinase-Streptokinase Embolism Trial: Phase 2. JAMA, 1974; 229: 1606-1613.

10. Goldhaber SZ. Thrombolytic therapy in venous thromboembolism: clinical trials and current indications. Clin Chest Med, 1995; 16: 307-320.

11. Addolorato G, Capristo E, Gessa GL et al. Long-term administration of GHB does not affect muscular mass in alcoholics. Life Sci, 1999; 65: L191-L196.

12. Tajima H, Murata S, Kamazaki T et al. Hybrid treatment of acute massive pulmonary thromboembolism: mechanical fragmentation with a modified pigtail catheter, local fibrinolytic therapy, and clot aspiration followed by systemic fibrinolytic therapy. AJR, 2004; 183: 589-595.

13. Kuo WT, Gould MK, Louie JD, Rosenberg JK, Sza DY, Hofmann LV. Catheter-directed therapy for the treatment of massive pulmonary embolism: systematic review and meta-analysis of modern techniques. J Vasc Interv Radiol, 2009; 20: 1431-1440.

14. Adam T, Arnaud P, Stavros K et al. Guidelines on diagnosis and management of acute pulmonary embolism: the task force for diagnosis and management of acute pulmonary embolism of the European Society of Cardiology. Eur Heart J, 2008; 29: 2276-2315.

15. Kucher N. Catheter embolectomy for acute pulmonary embolism. Chest, 2007; 132: 657-663.

16. Skaf E. Beemath A, Siddiqui T, Janjua M, Patel NR, Stein PD. Catheter-tip embolectomy in the management of acute massive pulmonary embolism. Am J Cardiol, 2007; 99: 415-420.

17. Eid-Lidt G, Gaspar J, Sandoval J, Santos FD et al. Combined clot fragmentation and aspiration in patients with acute pulmonary embolism. Chest, 2008; 134: 54-60.

18. Goldhaber SZ, Visani 1, De Rosa M. Acute pulmonary embolism: clinical outcomes in the International Cooperative Pulmonary Embolism Registry (ICOPER). Lancet, 1999; 353: 1386-1389. 